\title{
Educación ambiental en estudiantes del nivel superior ante la problemática ambiental en méxico
}

\section{Environmental education in higher level students in the face of environmental problem in mexico}

DOI: $10.46932 / \mathrm{sfjdv2n2-160}$

Received in: March 1st, 2021

Accepted in: May 30th, 2021

\author{
Dra. Catalina Vargas Ramos \\ Universidad Autónoma de Tamaulipa \\ E-mail: cvargasr@docentes.uat.edu.mx
}

M.G.A María Guadalupe Martínez Treviño

Universidad Autónoma de Tamaulipas

E-mail:mgmtrevino@docentes.uat.edu.mx

\author{
Dr. Alan León González Almaguer \\ Universidad Autónoma de Tamaulipas \\ E-mail: aleona@docentes.uat.edu.mx
}

\section{RESUMEN}

Los problemas ambientales existen desde una perspectiva global, por lo que no solo se limita a la disminución de especies vivas sino también al calentamiento global, el deterioro de la capa de ozono, la tala inmoderada de árboles, entre otros produciendo un daño irreparable en nuestro planeta (Avendaño, 2012).

Siendo una herramienta para frenarlos es la Educación ambiental, ya que tiene como objetivo dotar a los seres humanos de conocimientos y valores para participar conscientemente en la prevención y el cuidado del medio ambiente, para crear conciencia y poder dar soluciones a los problemas ambientales. Es por ello que la educación ambiental requiere de métodos y nuevos enfoques para afrontar los problemas del ambiente y que favorezcan las conexiones culturales, políticas y económicas para contribuir al desarrollo de la calidad de vida (Martínez, 2010)

Siendo el objetivo de esta investigación conocer el nivel de educación ambiental de los universitarios se realizó una investigación a 160 estudiantes, en un rango de edad de 19 y 24 años, por medio del método GREIC, el cual arrojo resultados favorables, con un nivel de educación ambiental alto, por lo que se puede notar que las acciones hasta ahora tomadas en el sector educativo van brindando frutos en la relación amigable que se debe tener con el medo ambiente, sin embargo se debe seguir trabajando para que esto continúe y así pasar de la educación al accionar de los jóvenes los cuales juegan un papel muy importante para hacer frente a la problemática ambiental.

Palabras clave: Problemas ambientales, Educación ambiental, Sector educativo

\footnotetext{
ABSTRACT

Environmental problems exist from a global perspective, which is why it is not only limited to the decrease of living species but also to global warming, the deterioration of the ozone layer, the excessive felling of trees, among others, causing irreparable damage to our planet (Avendaño, 2012).
} 
Being a tool to stop them is environmental education, since it aims to provide human beings with knowledge and values to consciously participate in the prevention and care of the environment, to create awareness and to provide solutions to environmental problems. That is why environmental education requires methods and new approaches to face environmental problems and that favor cultural, political and economic connections to contribute to the development of quality of life (Martínez, 2010)

Being the objective of this investigation to know the level of environmental education of the university students, an investigation was carried out to 160 students, in an age range of 19 and 24 years, by means of the GREIC method, which yielded favorable results, with a level of high environmental education, so it can be seen that the actions taken so far in the educational sector are bearing fruit in the friendly relationship that must be had with the environment, however, work must continue to ensure that this continues and thus go from education through the actions of young people, who play a very important role in dealing with environmental problems.

Keywords: Environmental problems, Environmental education, Educational sector

\section{INTRODUCCIÓN}

Numerosos autores consideran que los comienzos de la Educación Ambiental surgieron en la década de los 70, con el reconocimiento oficial de su existencia y de su importancia obtenida en la Conferencia de la ONU sobre el Medio Humano en Estocolmo, 1972, aunque existían referencias en documentos de años anteriores. En esta década, se constata la preocupación mundial por establecer estrategias educativas orientadas a la preservación del medio ambiente y por extensión, a una mejora progresiva en las condiciones de vida planetaria. Gracias a un incipiente movimiento conservacionista, se reclaman actuaciones políticas comprometidas con la prevención y solución de los problemas ambientales, una vez admitido el fracaso de la convicción de un crecimiento sin límites. De este modo, la Educación Ambiental en esos años se conoce como aquella educación que transfiere a los individuos la responsabilidad sobre las alteraciones del progreso, aunque sin cuestionar los principios económicos que los sustentaban (Avendaño, 2012).

Así mismo en la década de los 80, se produce una verdadera explosión social con la popularización del interés público sobre los temas ambientales. Comienza una etapa de transición en la que la percepción de la problemática ambiental se verá modificada por dos factores: las estrechas relaciones existentes entre los problemas ambientales y el modelo económico establecido en el orden internacional, así como la revelación del impacto ambiental global provocado por la acción humana, objetivado y expresado por la comunidad científica. En consecuencia, los procesos educativos relacionados con el medio ambiente ampliarán sus perspectivas más allá de las cuestiones ecológicas para ocuparse también del medio humano. La adopción de enfoques interdisciplinares y holísticos, ya considerada en los principios de la década anterior, se convierten en supuestos metodológicos cada vez más vinculados a la práctica. Por 
tanto, ante una crisis que es fundamentalmente cultural, se hace necesario desarrollar programas y actuaciones de carácter educativo que provoquen un cambio profundo en las escalas de valores y actitudes dominantes en la sociedad (Barroso, 2012

Por lo que hablar de la Educación ambiental no es un tema actual o que haya surgido recientemente, pero cabe mencionar que es un tema que ha cobrado más importancia en la actualidad, ya que en los últimos tiempos se han hecho más notorios los estragos en nuestro planeta; por lo que ha surgido la necesidad de orientar a las futuras generaciones para obtener una toma de conciencia de lo que está sucediendo con nuestro planeta por lo que la educación ambiental es necesaria para lograr una mejora en nuestra actualidad dirigida al medio ambiente (Sánchez \& Pontes, 2010).

Ya que la educación ambiental es considerada como un "proceso de aprendizaje que debe facilitar la comprensión de las realidades del medioambiente, del proceso socio histórico que ha conducido a su actual deterioro" (Sarango, Sánchez \& Landívar, 2016).

Así mismo Calixto, (2012) define la educación ambiental como el "proceso interdisciplinario para desarrollar ciudadanos conscientes e informados acerca del ambiente en su totalidad, en su aspecto natural y modificado; con capacidad para asumir el compromiso interdisciplinario para desarrollar ciudadanos consientes e informados acerca del ambiente en su totalidad, en su aspecto natural y modificado; con capacidad para asumir un compromiso de participar en la solución de problemas, tomar decisiones y actuar para asegurar la calidad ambiental".

Por lo que la importancia de la educación ambiental es conseguir que la sociedad adquiera los conocimientos y valores para participar conscientemente en la prevención y el cuidado del medio ambiente. No solo abarca aspectos ecológicos; trata en gran parte de las responsabilidades que deben de tener la sociedad para ser capaces de hacer cambios y que garanticen un desarrollo sustentable como tener conciencia para solucionar problemas ambientales .Es por ello que la educación ambiental requiere de métodos y nuevos enfoques para afrontar los problemas del ambiente y que favorezcan las conexiones culturales, políticas y económicas para contribuir al desarrollo de la calidad de vida (Martínez, 2010)

Entendiendo así que los problemas ambientales existen desde una perspectiva global la cual se ha hecho más variada, por lo que no solo se limita a la disminución de especies vivas sino también a otros aspectos como lo son el calentamiento global, el deterioro de la capa de ozono, la tala inmoderada de árboles, al igual que la ruina del capital histórico-cultural por lo que está produciendo un daño irreparable en nuestro planeta (Avendaño, 2012).

Mientras que la problemática ambiental se debe a que la mano del hombre desempeña un papel importante en nuestro planeta ya que a causa del mal uso de los recursos ya no es certero que el ambiente pueda seguir teniendo la misma capacidad de sostener a las futuras generaciones a causa del mal uso de 
los recursos ambientales. Un ejemplo claro de esto es el deterioro de bosques tropicales y templados en el mundo ya que la mitad de ellos han desaparecido y más de la mitad de las pesquerías en el mundo se han acabado o se utiliza su capacidad de manera desmedida y de la misma manera las tierras utilizadas para cultivos se encuentran muy dañadas por lo que el conjunto de todos estos problemas causa lo que se conoce como cambio climático (Sosa, Márquez, Eastmond, Ayala \& Arteaga, 2010).

A través de esta problemática surgió la necesidad de apoyar a las futuras generaciones en la toma de conciencia de los graves problemas ambientales que están enfrentando día con día por lo que en la actualidad se toma como un tema de interés para la educación de las nuevas generaciones que serán las que enfrentarán estos complicados retos. Por lo que debe ser importante recalcar la necesidad de aumentar la educación ambiental y seguir adelante en la búsqueda de una cultura medioambiental de manera mundial (España y Prieto, 2010).

Por lo tanto, la problemática ambiental tiene que ser encaminada a los seres humanos en general ya que es un problema que debe de importarle a todos lo cual debe de dar como resultado cambios en la calidad de vida, en el comportamiento de las personas y las relaciones del ser humano que acarreen una mejora hacia la manera de vivir del ser humano y el planeta es por ello que surgió la formulación de un compromiso de prevención en donde se enfocan siete corrientes ambientales las cuales aparecieron hace más de 40 años con la finalidad de dar a conocer a las personas lo que está ocurriendo con el medio ambiente (Martínez, 2010).

Por lo que, Avendaño, (2012) menciona las siete corrientes de la educación ambiental consideradas como centro de esta: naturalista, conservacionista, resolutiva, sistemática, científica, humanística y moralética.

La corriente naturalista tiene 3 enfoques el primero es el tener el objetivo de conocer más sobre la naturaleza, de lo que tiene la misma y lo que se puede aprender de ella mediante la observación, el segundo enfoque se basa mayormente en las experiencias que se obtienen mediante la naturaleza y de la misma manera que el primer enfoque se obtiene un aprendizaje y por último el tercer enfoque de esta corriente va más dirigida al arte, lo espiritual y lo efectivo en donde puede surgir un cambio a favor de lo que conlleva la naturaleza y su relación (Pérez, 2016).

Luego, Avendaño, (2012) menciona aspectos relevantes sobre la misma corriente naturalista, esta es una de las iniciales ya que se posiciona en relación con la naturaleza y el medio ambiente en un punto de vista cognitivo, experimental afectivo y artístico, de donde se toma el conocimiento y se entra en contacto con este medio natural, (educación al aire libre). La idea de aceptación de la corriente naturalista acepta el valor de la misma naturaleza, más allá de los bienes, riquezas y medios de subsistencia, que esta pueda brindar. Afirma que el conocimiento desata en la relevancia de tomar en cuenta la naturaleza como 
educadora y como medio relacionado de un aprendizaje eficiente para el entendimiento de los derechos inherentes del medio ambiente.

Seguidamente Sauve, (2013) afirma que en cuanto la corriente conservacionista, están conjuntas las ideas dirigidas hacia la protección y cuidado de los recursos, tanto en posturas cualitativas como en cuantitativos; y en ella se expone una preocupación por la administración ambiental, siendo integrante para de la educación comunitaria la cual se representa en forma de educación ambiental.

En cuanto a la corriente resolutiva, se da a conocer la gravedad, el peligro y el crecimiento y ampliación de la problemática ambiental, esta corriente asume la noción central establecida por la UNESCO en su programa internacional de educación ambiental, esta corriente enseña un modelo de enseñanza visualizado en el logro de capacidades para la resolución de las problemáticas, en donde se sostiene a la educación ambiental como el principio de estudio de la problemática ambiental, este también tomando en cuenta partes biofísicos y sociales, llegando al entendimiento de una situación problema, la investigación de esta, el reconocimiento de la búsqueda de las respuestas, la evaluación de soluciones ideales , para lograr de este modo, tener un entendimiento adecuado de las problemáticas ambientales en las que se encuentra dentro nuestro medio ambiente de este modo llegando de igual manera a la educación ambiental, ya que se tiene en cuenta los conocimientos necesarios para la determinación de valores de riesgo para el medio que nos rodea (Avendaño,2012).

Por otra parte Covas, (2018) menciona sobre la corriente sistemática que la educación ambiental tiene en cuanto a los principios ecológicos y que debe inducir a el cambio comunitario y a el cambio de la conciencia humana, todos las problemáticas medioambientales están constituidas indispensablemente por un sistema, al considerarlo como un todo organizado ,constituido por partes que interactúan entre sí, también el medio ambiente se constituye por una parte sistemática, donde los elementos de dicho sistema están integrados en el medio físico, biótico, económico y sociocultural., de tal forma que el cambio que se creen en algunos de estos elementos afecta a los demás y como la unificación entre ellas conlleva al desarrollo, sin una de estas no se puede producir el conocimiento exacto de la educación ambiental dada la relevancia y la conexión entre las mismas.

Mientras que la corriente científica tiene como objetivo el afrontar detalladamente y de manera realista los problemas ambientales, esto con el objetivo de reconocer de una forma más concreta las relaciones que existe en una acción donde siempre surge una reacción. En esta corriente la educación ambiental está ampliamente asociada al entendimiento y la capacidad que se tiene para comprender las ciencias ambientales. En esta corriente se incorporan etapas de un proceso científico las cuales son la exploración y observación del medio, la identificación de fenómenos, y la realización de un plan para resolver un problema planteado (Avendaño, 2012). 
En cuanto a la corriente humanista, esta se centra en la parte humana con el medio que lo rodea, es decir, la relación entre el medio ambiente y cultura que comprende las dimensiones históricas , políticas y económicas a modo de patrimonio, pues las construcciones y ordenamientos humanos son parte presencial de la alianza entre la creación humana y los materiales de la naturaleza, hacen enfoque en un modelo que busca un medio ambiente como un medio de convivencia social, donde se exhorta a una búsqueda de visualizaciones libres y dirigidas a tocar la parte sensible y efectiva del ser humano, que toman como planeación ideas de investigación las cuales se basan en encontrar la comprensión atinada de la realidad del medio de vida que se desarrolla en nuestro entorno, de tal modo que las personas puedan afrontar las problemáticas ambientales de una forma sensible, emocionalmente, relacionando el aprendizaje, con el medio ambienten de esta forma llegando a la educación ambiental sin que estas personas lo hayan tomado como estudio si no como conocimiento vivido por ellos mismos (Maldonado,2010).

Por último, la corriente moral-ética es muy utilizada por los docentes como raíz de la relación del medio ambiente con el hombre, destacando que el actuar debe estar presente en un conjunto de valores para crear un vínculo entre ellos. Como modelo educacional, hace hincapié en la formación moral de los educados, así como también el razonamiento social. El objetivo de esta corriente es el poder tratar las situaciones y la toma de decisiones mediante estrategias como lo son, la presentación y análisis de un caso con sus componentes sociales y morales, la elección de una resolución acorde a una conducta apropiada, y sustentar esta elección, con la meta de crear una discusión donde se intercambien ideas éticas que den soluciones correctas ante la problemática ambiental adoptada (Avendaño, 2012)

Con lo anterior se refuerza el conocimiento de las bases de la educación ambiental o parte de ellas que las hace tener un valor más importante ya que se consolido como la parte fundamental del cambio social, a partir de planes para determinar responsabilidades ambientales en pro de todos los países, ya que es conocida como una herramienta necesaria para aumentar el beneficio de las relaciones humanas con el medio que los rodea. En lo que respecta a la solución de la problemática ambiental que en él se presente, ya que es ahí donde se introduce la temática de la E.A. a los sistemas educativos, habiendo más notoria la urgencia del cambio de actitudes, incluir conocimientos y criterios, tomando como parte inclusiva a las comunidades, dirigiéndolas a las búsquedas de salidas y respuestas para las problemáticas ambientales existentes a nivel mundial (Martínez, 2012).

A lo cual Avendaño (2012), menciona que todas la corrientes tienen suma importancia debido a que todas estas deben proporcionar y debe generar un cambio de pensamiento, tomando en cuenta la participación ciudadana de las personas y la gestión del ambiente para que de este modo se pueda obtener trabajos apropiados en el entorno, cabe mencionar que la educación habitual no ha podido ser muy 
adecuada ya que no se obtienen cambios significativos y verdaderos en el actuar de las personas y sus acciones socioambientales. Es por ello que se requiere dar origen a nuevas corrientes educativas que generen nuevos cambios en favor del ambiente con el propósito de generar un cambio de actitud en las actuales y nuevas generaciones.

No obstante García, (2012) menciona que cada corriente educativa se basa en la concepción del aprendizaje y se sostiene en la persona que lo desarrolla por ello menciona que "Hay que fomentar uma pedagogía de la inquietud: la pedagogía de situar a los hombres y mujeres ante su responsabilidad, forjar actitudes, contribuir a que cada uno sea soberano de sí mismo. Soberano crítico guiado por estrellas altas que no se compren ni se vendan. Buscando aprendizajes comunitarios que liberen y hagan aflorar lo mejor de nuestro saber, teniendo como libro de texto la vida cotidiana. Dando a entender que cada persona es responsable de llevar a cabo cada una de las corrientes de forma responsable del mismo modo de forma directa o indirecta pero siempre basándose de un principio de partida y encaminada hacia la educación ambiental.

Es por ello que en la actualidad se hace mayor énfasis en la educación ambiental ya que esta ayuda a que los seres humanos obtengan una conciencia más amplia de la situación por la que se está viviendo en el planeta, por lo que la educación ambiental ayuda a fomentar la capacidad de interpretar las problemáticas que se viven en la actualidad para poder llegar a realizar un cambio positivo para el medio ambiente y así contribuir de manera efectiva una mejora al planeta (Martínez, 2010).

Lo antes mencionado se dirige hacia un nuevo orden en donde abarca lo social, así como lo cultural en relación con el ambiente para mejorar las condiciones de vida aumentando la calidad de la misma conforme a los hábitos que se generen ambientalmente y la cultura que se va arraigando en el proceso de la educación ambiental (Avendaño, 2012).

Es por ello por lo que Calixto (2012) menciona finalmente que al adquirir responsabilidades ambientales se crea una nueva conciencia denominada "conciencia planetaria" la cual se enfoca en la capacidad humana que se adquiere para analizar y reflexionar sobre los hechos que ocurren en torno al ambiente. En conclusión, se puede deducir que la educación ambiental es un mar abierto a cuestiones en donde las acciones y eventos donde los seres humanos ayudan a alentarlos y generar los valores en las personas como los son la participación ciudadana, la madurez, disciplina y compañerismo en pro del medio ambiente.

Así mismo distintos autores han indagado sobre este tema de Educación Ambiental encontrándose diferentes estudios como los que a continuación se mencionan.

Isaac, R., et al, en el año 2011 realizo un estudio cuantitativo y cualitativo a estudiantes de bachillerato en Campeche para saber el nivel de educación ambiental entre los integrantes de dicho plantel. 
Realizando una encuesta diseñada para evaluar la educación y cultura ambiental que constaba de 15 reactivos de los cuales contenían respuestas de opción múltiple y solo 1 reactivo contenía la respuesta correcta por lo que las opciones restantes eran erróneas. Los resultados arrojaron que la comunidad estudiantil se encuentra en una situación pobre ambientalmente hablando, ya que el resultado que obtuvieron fue tomado en cuenta el valor de ponderación que va del 1 al 10, por lo que al tener una calificación de 5.6 de promedio, se toma como calificación reprobatoria según la Secretaria de Educación Pública (SEP).

Mientras que Vargas et al durante el año 2011 realizo una encuesta basada en 30 reactivos la cual se aplicó en los salones de una unidad académica, para recabar la información y darles a conocer la manera correcta en cómo responder dicha encuesta. La duración de la encuesta fue entre 15 y 25 minutos, fue de manera anónima y la colaboración de los alumnos fue de manera opcional. Donde el 45,6 \% de los encuestados perteneció a la carrera de Psicología, sucesivo del 28,1\% para los estudiantes de la carrera de Seguridad Salud y Medio Ambiente y para finalizar el 26,3\% pertenece a la carrera de Enfermería, tomando en cuenta que la cantidad de alumnos encuestados fueron 200. De acuerdo a la encuesta realizada al total de alumnos encuestados se obtuvieron los siguientes resultados: el $2.1 \%$ son consumistas conscientes y el $97.9 \%$ no llevan a cabo un buen cuidado del medio ambiente de lo que se podría interpretar que de los 200 alumnos encuestados 196 no son conscientes del cuidado del ambiente por lo que solo 4 son conscientes del cuidado del medio ambiente.

Así mismo Espejel, Flores \& Castillo en el año 2014 llevaron a cabo una investigación sobre la educación ambiental en la preparatoria CBTis 212 desde el punto de vista de género en la ciudad de Tlaxcala, México, teniendo como finalidad describir como la comunidad estudiantil según su género pueden proponer acciones a favor del medio ambiente; la investigación se realizó mediante la metodología cualitativa a través de cuestionarios donde se tenían que realizar programas ambientales, fueron 80 estudiantes de los cuales 45 fueron hombres y 35 mujeres los que realizaron actividades ambientales. Para conocer y determinar cómo estudiantes de ambos sexos realizan acciones en favor del ambiente, se conformaron un total de 12 equipos del cuarto semestre de las especialidades de informática y mecatrónica del turno matutino y vespertino, para que diseñaran programas ambientales (PA). Con la finalidad de conocer, desde la perspectiva de género las acciones y objetivos que se proponen en su PA para mitigar el deterioro ambiental de su escuela. Lo que les dio como conclusión se observó que la buena práctica de programas ambientales contribuye a que los alumnos propongan acciones por ellos mismos para el cuidado del medio ambiente y donde se observa que las mujeres tienen mayor cuidado al no desperdiciar agua y una mejor visión respecto a la contaminación visual. 
A lo que Vargas et al en el año 2010 realizo la comprobación de la conciencia ambiental (CA) que muestran los estudiantes de la licenciatura en Enfermería Básica y el elemento social con mayor Conciencia Ambiental (CA) con un total de 119 estudiantes entre los 2 turnos matutino y vespertino indican que el 52.94\% tienen actitud ambientalista, mientras que el $47.06 \%$ tiene conocimiento ambiental.

Así mismo, Vargas, Medellín \& Vázquez en el 2011, aplicaron un estudio de actitudes ambientales a los estudiantes de nivel superior en México la muestra fue basada en la escala de Likert compuesta por 11 ítems. Midiendo la variable de actitud ambiental la cual está compuesta de cuatro formas 1 consumista derrochador,2 consumista consienta,3 ecologista bien encaminado,4 ecologista cuidadoso de la madre tierra, donde sus resultados confirman que el $75.3 \%$ son ecologistas bien encaminado seguido del $24.7 \%$ consumista consiente por lo cual quiere decir que los alumnos poseen conocimientos sobre la conservación y el cuidado del medio ambiente y que no existen diferencias entre las actitudes del alumno respecto a la carreras que están realizando.

\section{MATERIALES Y MÉTODOS.}

La metodología utilizada para llevar a cabo esta investigación es de tipo descriptiva- Explicativa, y fue aplicada a un total de 160 estudiantes del nivel superior siendo 80 hombre y 80 mujeres los cuales oscilaban entre los 19 y 24 años de edad.

El método con el que se llevó a cabo la investigación, recopilo información por medio del instrumento llamado GREIC (de la universitat de iles belears) donde, la adaptación del cuestionario presento un instrumento con un total de 50 items tipo Likert, cuyas respuestas van desde el No sé, No, En parte no, En parte si y Si, donde "No se” representa la puntuación más baja en la evaluación y "Si” la

puntuación más alta, cuyo principal objetivo es medir el nivel de educación ambiental en los alumnos evaluados con preguntas relacionadas a situaciones ambientales que se presentan en el día a día, con un nivel de complejidad bajo.

En cuanto a los niveles de educación ambiental que muestra el instrumento utilizad, indica en la tabla 1 que la puntuación de 156-104 es una educación ambiental alta, de 103-52 tienen un nivel de educación ambiental media y de 51-0 un nivel de educación bajo (Ver tabla 1).

Tabla 1.- Niveles de Educación Ambiental

\begin{tabular}{ll}
\hline TABLA 1.- & NIVEL DE EDUCACIÓN \\
AMBIENTAL & \\
\hline $156-104$ & Educación Alta \\
\hline $103-52$ & Educación Media \\
\hline $51-0$ & Educación Baja
\end{tabular}


Para la obtención y clasificación de los datos recabados se utilizó el paquete estadístico SPSS versión 23.

\section{RESULTADOS}

De acuerdo a la información reunida para encontrar el nivel de educación con el que cuentan los estudiantes de nivel superior se obtuvieron los siguientes resultados, el 69\% de los estudiantes de nivel superior cuentan con una educación ambiental alta, mientras que el 21\% tiene educación ambiental media y solo un $10 \%$ cuenta con una educación ambiental baja (Ver grafica 1).

Gráfica 1.-Education ambiental en estudiantes universitarios.

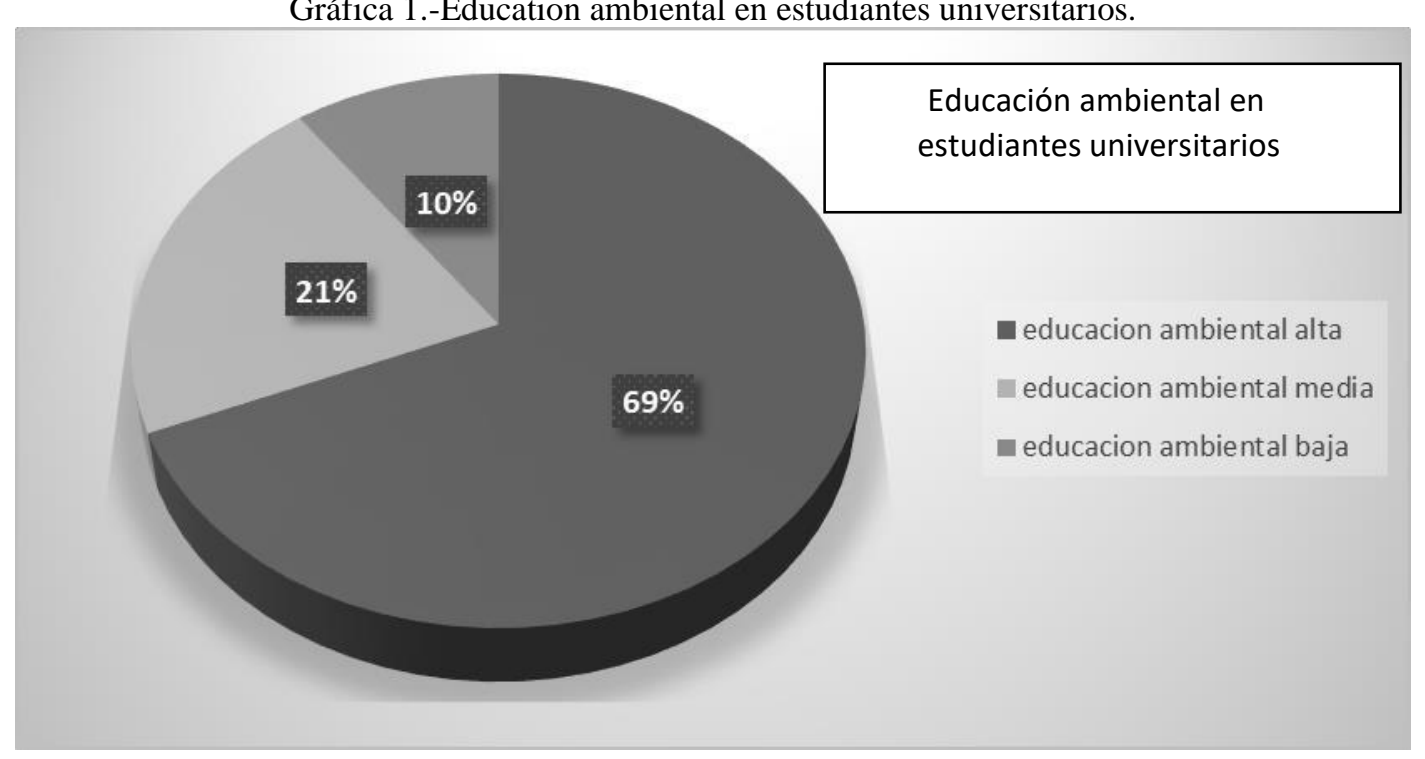

En la siguiente grafica se muestran los porcentajes obtenidos por sexo, en primer lugar, la gráfica muestra que el $65 \%$ de los hombres tienen educación ambiental alta, mientras que el 22\% cuentan con educación ambiental media y el 13\% educación ambiental baja (Ver grafica 2). 
Gráfica 2.-Educación Ambiental en hombres.

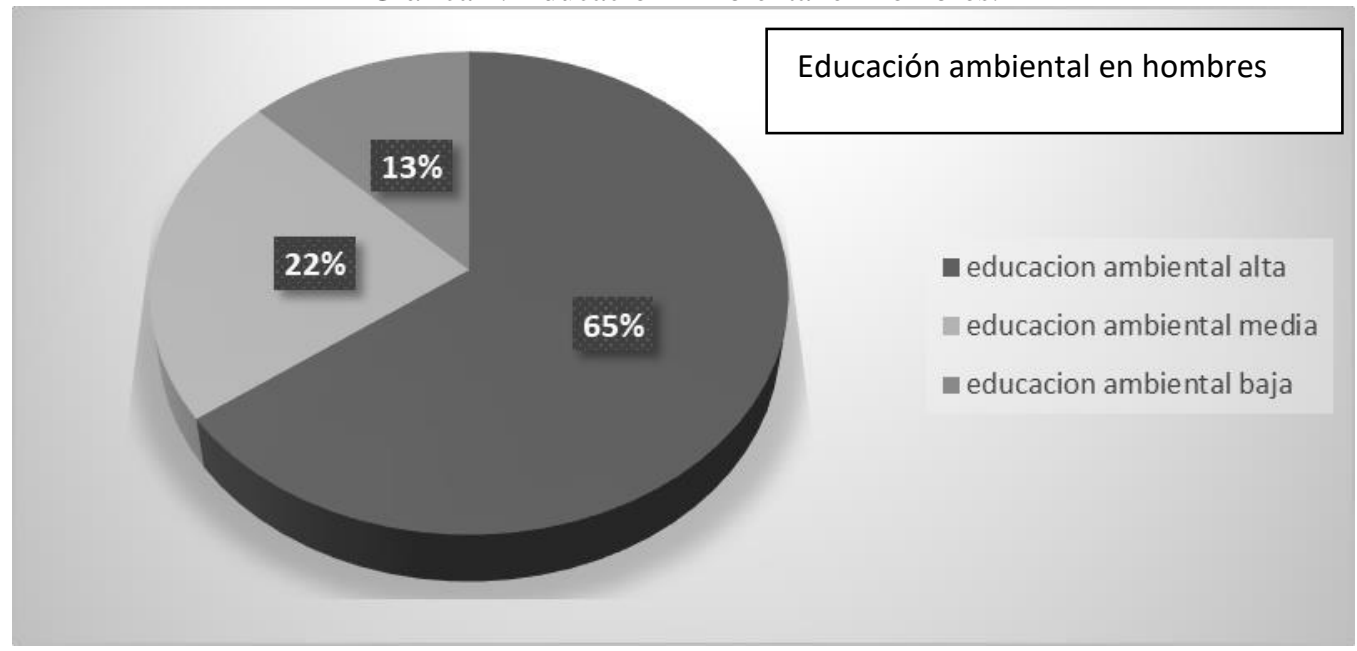

En cuanto a las mujeres los resultados indican que el $72 \%$ cuenta con educación ambiental alta, el $20 \%$ educación media y el $8 \%$ educación ambiental baja (Ver gráfica 3).

Gráfica 3.-Educación ambiental en mujeres.

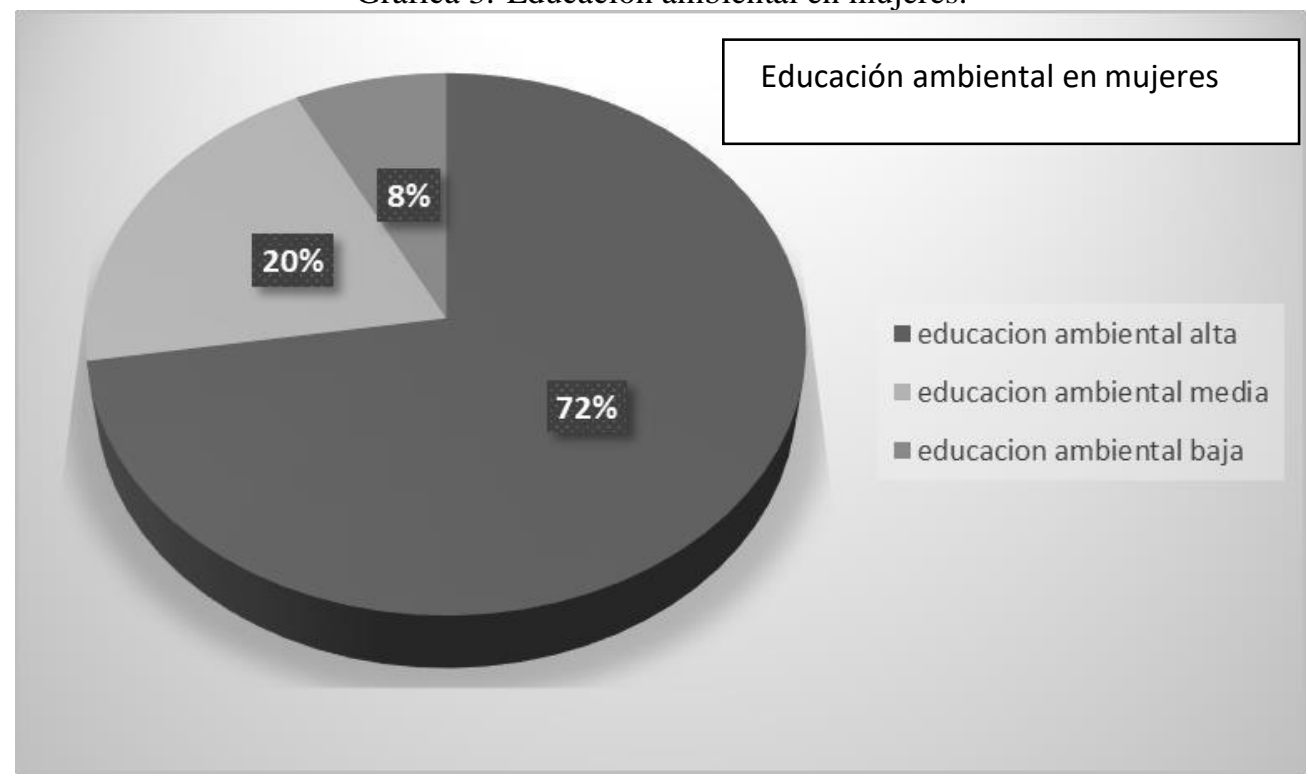

Así mismo se presenta la siguiente resultados en cuanto a los porcentajes obtenidos en estudiantes universitarios por estado civil, en el cual se puede apreciar que el nivel de educación alta es de $56 \%$ el cual corresponde a estudiantes solteros, mientras que el nivel de educación alta en casados es de un 14\% por lo que nos indica que los solteros cuentan con mayor educación ambiental; en cuanto a la educación ambiental media los porcentajes refieren que el $20 \%$ de los solteros cuentan con educación ambiental media, del mismo modo se obtuvo los resultados de la educación ambiental baja en donde aprecia que un $8 \%$ de los solteros cuenta con ella y solo un $2 \%$ que equivale a los casados cuentan con educación ambiental baja (ver grafica 4$)$ 
Gráfica 4.- Educación ambiental por estado civil.

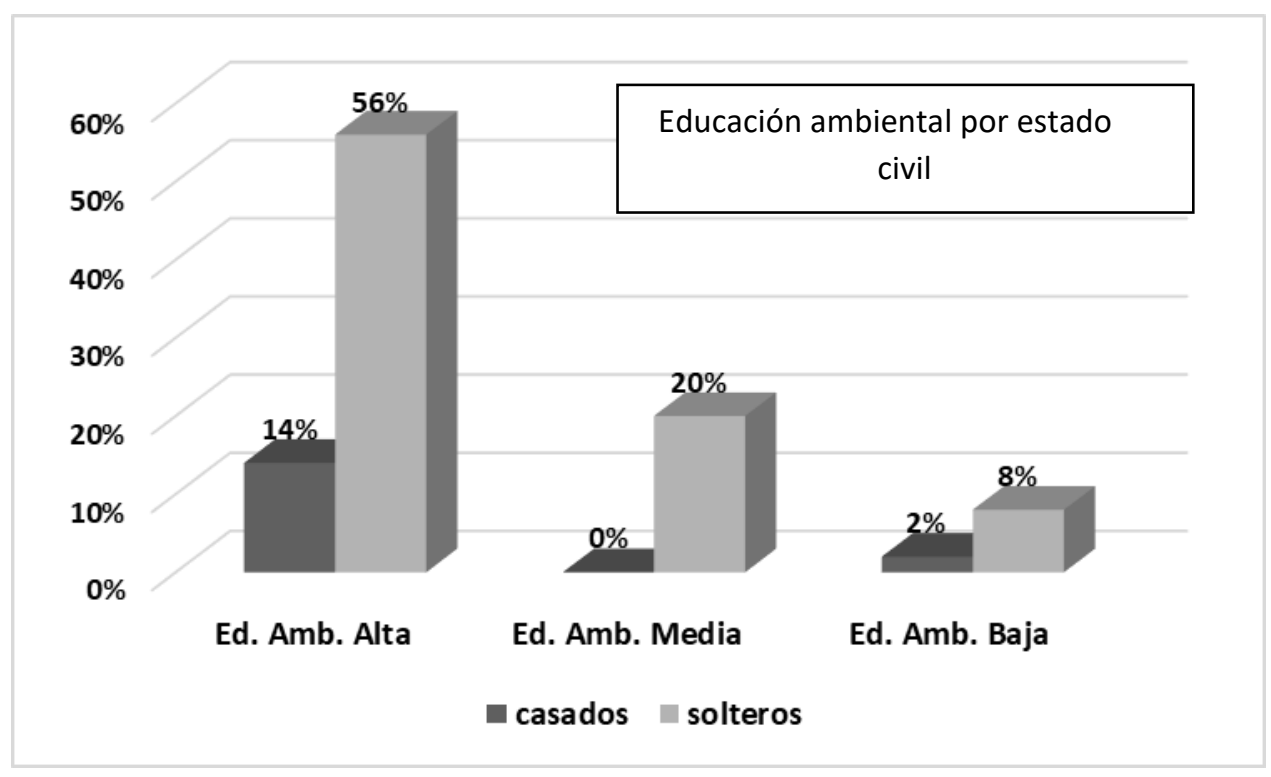

Conforme a los resultados que se obtuvieron por rango de edad, se encontró que en las edades de 19 a 20 años el $20 \%$ cuenta con un nivel de educación ambiental alta, así mismo el 5\% cuentan con un nivel medio y un $4 \%$ con un nivel de educación baja, mientras que los de rango de edad de 21 a 22 años el $26 \%$ corresponde a un nivel de educación alta y el 5\% pertenece a un nivel medio y el otro 5\% es baja. De igual manera los estudiantes de 23 a 24 años cuentan con un 23\% de educación ambiental alta, un 10\% con educación ambiental media y el $2 \%$ con un nivel bajo, (ver grafica 5).

Gráfica 5.- Educación ambiental por rango de edades.

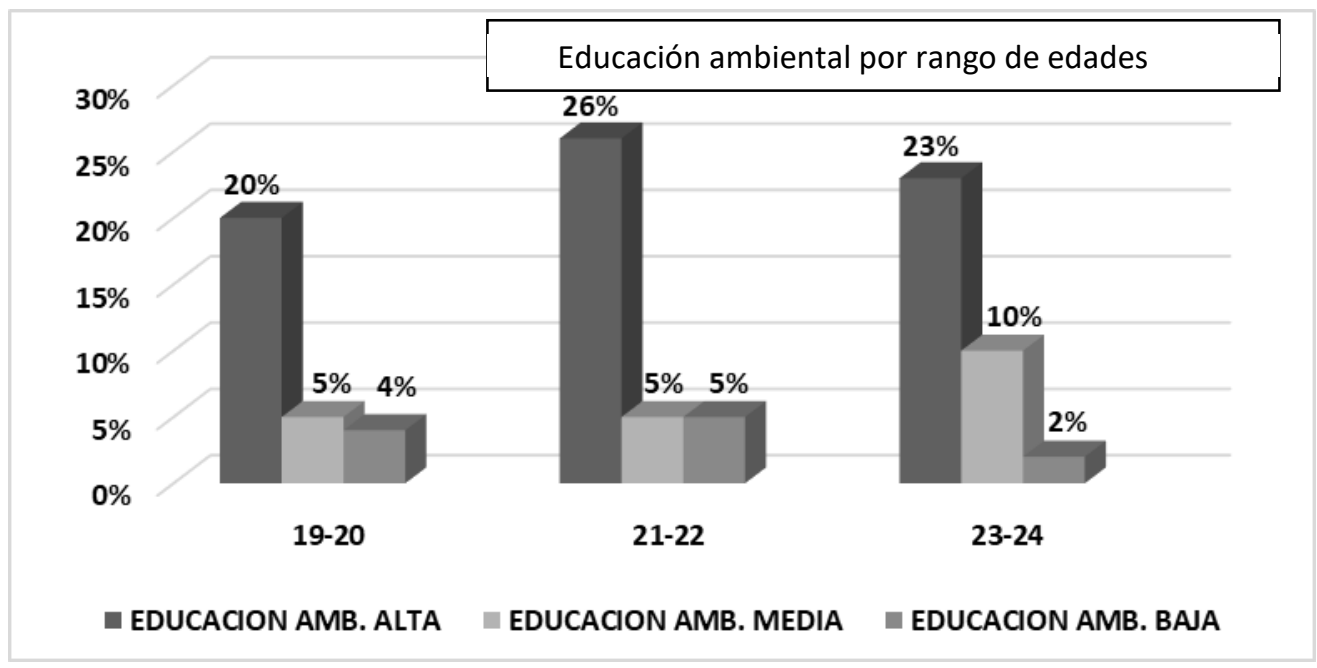

\section{DISCUSIÓN}

De acuerdo a los resultados obtenido en la investigación del 2011 realiza por (Vargas et al) los resultados fueron similares ya que en ambos los niveles de educación ambiental prevalecieron en altos, 
cabe señalar que, aunque la investigación fue en la misma institución la generación a quien se le realizo fue distinta por lo que se deduce que se sigue trabajando día a día para que los resultados se mantengan.

En cuanto a los resultado que se pudieron comparar por estado civil se encontró que los estudiantes del sexo femenino cuentan con un porcentaje mayor de nivel de educación ambiental comparado al porcentaje obtenido en estudiantes de sexo masculino y de acuerdo con la investigación realizada por (Espejel, flores \& castillo 2014) los cuales tenían como finalidad describir como la comunidad estudiantil en la ciudad de Tlaxcala proponían acciones a favor del medio ambiente se encontró que las mujeres tienen mayor cuidado con el medio ambiente por lo que los resultados de ambas investigaciones son similares.

Sin embargo, si se compara con los resultados de la investigación que realizo (Issac, R et al 2011) en donde obtuvo que la comunidad estudiantil tiene un indicador pobre hablando ambientalmente, difiere ya que la comunidad estudiantil que participo en nuestra investigación prevaleció en niveles altos de educación ambiental

\section{CONCLUSIONES Y RECOMENDACIONES}

Después de determinar el resultado de cada estudiante de este investigación y comparados con los estudios previos se puede concluir que los estudiantes de nivel superior cuentan con un nivel de educación ambiental alta de acuerdo a los resultados que se obtuvieron anteriormente ya que el $69 \%$ obtuvo un índice alto en educación ambiental, el $21 \%$ educación ambiental media y solo el 10\% cuentan con educación ambiental baja, sin embargo se recomienda que se sigan implementando estrategias para generar educación ambiental en los estudiantes que si bien es cierto que se va por buen camino un hace falta que se sumen más estudiantes al entendimiento del cuidado del medio ambiente y al impacto que se generar con sus acciones hacia este.

En cuanto a los resultados que se obtuvieron por rango de edad se observa que el nivel de educación que prevaleció fue el alto obteniendo el rango de 19 a 20 años el 20\%, el rango de 21-22 años el 26\% y el rango de 23-24 años el 23\% siendo esto favorable en cuanto al papel que juega la inclusión de temas ambientales en los programas educativas, sin embargo aún no seguimos encontrando aunque con mínimos porcentajes la educación ambiental baja lo cual si se sigue trabajando podría remolcarse a sumar estos porcentajes a los niveles altos de educación.

Así mismo al analizarse los resultados por estado civil se encontró que el porcentaje obtenido en cuanto a los niveles de educación ambiental predomino los solteros con un $56 \%$ y en los casados con un $14 \%$. 
Como se puede notar los resultados para esta investigación en su mayoría son favorables hacia los niveles altos de educación ambiental justificado por la conciencia que se ha tomado sobre este temas debido a los problemas y crisis ambientales que se viven hoy en día, sin embargo es algo en lo que se

tiene que seguir trabajando, tomando en cuenta que esta investigación fue realizada a estudiantes del nivel superior se puede decir que las acciones que se han tomado desde la educación preescolar van por buen camino, ya que el fin es cambiar el pensamiento del ser humano para que cada acción y decisión que tome a lo largo de su vida sea pensando en la relación amigable que debe existir hacia el medio ambiente y que al final solo se tiene este para vivir.

Por lo tanto podemos recomendar con base a los resultados que se obtuvieron que esta investigación puede ser de gran relevancia para futuras investigaciones de la misma rama como base para proponer mejoras ya que de acuerdo a que los resultados fueron favorables se puede recomendar que sigan incluyendo materias dirigidas al cuidado y preservación del medio ambiente como materias básicas en el plan de estudios lo cual ayuda a mejorar la calidad de vida y la concientización que logran los estudiantes ante la problemática que surge hoy en día con el medio ambiente, de igual manera se recomienda incluir asignaturas desde el nivel de educación básico ya que de esta manera es más sencillo que se genere una toma de conciencia y un hábito en el cuidado del medio ambiente para seguir mejorando la calidad de vida de futuras generaciones tomando en cuenta los diferentes beneficios que se pueden ir obteniendo.

Por otra parte, se debe de tener en cuenta el poner en práctica los conocimientos adquiridos para fortalecer la cultura preventiva en materia medioambiental con prácticas, formación de grupos ambientales, dinámicas en donde los estudiantes puedan tener contacto directo con la naturaleza con el fin de obtener una mayor educación ambiental o un mayor respeto hacia el medio ambiente. 


\section{REFERENCIAS BIBLIOGRÁFICAS.}

Avendaño, W., (2012). La educación ambiental como herramienta de la responsabilidad social. Revista luna azul. No. 35. P.p. 94-115. Disponible en: www.scielo.org.co/pdf/luaz/n35/n35a07.pdf

Barroso, V., (2012). ¿Un conflicto ambiental o de territorio? Revista Letras verdes. $N^{\circ} 11$. pp. 112-122. Disponible en: https://revistas.flacsoandes.edu.ec/letrasverdes/article/view/919/880

Calixto, R., (2012). Investigación en educación ambiental. Revista mexicana de investigación educativa.

No. 55. Vol. 17. P.p. 1019-1033. Disponible en: www.scielo.org.mx/pdf/rmie/v17n55/v17n55a2.pdf

Covas, O., Guerra, M. \& Santos, I., (2018). Nuevas perspectivas para la educación ambiental en la educación de adultos. Varona, Revista Científico-Metodológica. No. Especial. pp. 1-13. Disponible en: http://revistas.ucpejv.edu.cu/index.php/rVar/article/view/446/676

Espejel, A., Flores, A., \& Castillo, I., (2014). Educación ambiental en el nivel medio superior, desde la perspectiva del género. Revista electrónica educare. No. 3. Vol. 18. pp. 17-38. Disponible en: https://www.redalyc.org/pdf/1941/194131745002.pdf

García, M., (2012).la educación ambiental en 1 formación docente inicial en México. Revista pesquisa en educación ambiental. $\quad \mathrm{N}^{\circ} 2$. Vol. 2.pp. 80-92. Disponible en: https://www.researchgate.net/publication/323387325_LA_EDUCACION_AMBIENTAL_EN_LA_FO RMACI ON_DOCENTE_INICIAL

González, E., \& Arias, M., (2009). La educación ambiental institucionalizada: actos fallidos y horizontes de posibilidad. Perfiles educativos. No. 31, Vol. 124. pp. 58-68. Disponible en: http://www.scielo.org.mx/scielo.php?script=sci_arttext\&pid=S0185-26982009000200005

López, R., Bastida, D., (2018). La importancia de la educación ambiental no formal en el medio rural: en el caso de Palo Alto, Jalisco. Revista Diálogos sobre Educación. No. 16. P.p. 1-19 disponible en: http://www.scielo.org.mx/pdf/dsetaie/v9n16/2007-2171-dsetaie-9-16-00004.pdf

López, E., Cruz, C., Rodríguez, A., (2013). Educación para la sustentabilidad. Una experiencia chontal. Revista Horizonte Sanitario. No. 3. Vol. 12. Disponible en: http://revistas.ujat.mx/index.php/horizonte/article/view/84/839

Maldonado, T., (2010). Modelo de evaluación y acreditación de los centros de educación y cultura ambiental. Horizonte sanitario. No. 2, Vol. 9. pp. 42-47. Disponible en: https://www.redalyc.org/pdf/4578/457845135003.pdf

Martínez, R., (2010). La importancia de la educación ambiental ante la problemática actual. Revista electrónica educare. No. 1. Vol. 14. pp. 97-11. Disponible en: https://dialnet.unirioja.es/servlet/articulo?codigo $=4780944$

Medina, I., (2014), La investigación en educación ambiental en américa latina: un análisis bibliométrico. Revista colombiana de educación. $\mathrm{N}^{\circ} 66 . \quad$ pp.19-72. Disponible en: http://www.redalyc.org/articulo.oa?id=413635257003

Montaño, F., Cervantes, A., Gutiérrez, M., \& Zarate, M., (2012). La educación ambiental en México ante la crisis ambiental Revista Vinculando. $\mathrm{N}^{\circ}$ 1. pp. 2-14. Disponible en: http://delos.eumed.net/14/sbco.pdf 
Pérez, M., (2016). Corriente Naturalista De La Educación Ambiental analizada bajo el contexto colombiano, como una oportunidad para el desarrollo sostenible. Revista: Atlante $\mathrm{N}^{\circ} 1$. Vol.1. Disponible en: www.eumed.net/rev/atlante/2016/03/naturalista.html

Prieto, T., \& España, E., (2010). Educar para la sostenibilidad. Un problema del que podemos hacernos cargo. Revista Eureka. $\mathrm{N}^{\circ}$ Extraordinario. Vol.7. pp. 216-229. Disponible en: https://rodin.uca.es/xmlui/bitstream/handle/10498/8936/4_Prieto_Espa\%FDa_2010.pdf?sequence=1 Quiva, D., \& Vera, L., (2010). La educación ambiental como herramienta para promover el desarrollo sostenible. TELOS, Revista de estudios interdisciplinarios en ciencias sociales. Vol.12 N³. pp. 378-394. Disponible en: www.redalyc.org/html/993/99317168008/

Sánchez, F., \& Pontes, A., (2010). La comprensión de conceptos de ecología y sus implicaciones para la educación ambiental. Revista Eureka sobre Enseñanza y Divulgación de las Ciencias. Vol. 7, $\mathrm{N}^{\circ}$ Extraordinario. P.p. 271-285. Disponible en línea: https://rodin.uca.es/xmlui/bitstream/handle/10498/8942/8_Sanchez_Pontes_2010.pdf?sequence=1\&isAl lowed $=\mathrm{y}$

Sarango, J., Sánchez, S., \& Landívar, J., (2016). Educación ambiental ¿Por qué la historia?. Revista scielo. No8. Vol.3. P.p.184-187. Disponible en: www.scielo.sld.cu/pdf/rus/v8n3/rus25316.pdf

Sauvé, L., (2013). Educación ambiental y ecociudadania. Dimensiones claves de un proyecto políticopedagógico. Revista científica. $\mathrm{N}^{\circ} 18$. P.p.12-23. Disponible en: https://revistas.udistrital.edu.co/ojs/index.php/revcie/article/view/5558

Sosa, S., Isaac, R., Eastmond, A., Ayala, M., \& Arteaga, M., (2010). Educación superior y cultura ambiental en el sureste de México. Trópico húmedo. No. 26. Vol. 1. P.p. 33-49. Disponible en: www.scielo.org.mx/pdf/uc/v26n1/v26n1a3.pdf

Vargas, C., Medellín, J., Vázquez, L., \& Gutiérrez, G., (2011). Actitudes ambientales en los estudiantes de nivel superior en México. Revista Luna Azul. N³3. P.p. 31-35. Disponible en: www.scielo.org.co/pdf/luaz/n33/n33a04.pdf

Vargas, C., Vázquez, L., Gutiérrez, G., Vargas, A., \& Fernández, C., (2010). Conciencia ambiental en estudiantes de enfermería básica. Revista Universitaria de investigación y dialogo académico. ํ⒊ Vol.6. P.p.18-25. Disponible en: https://www.researchgate.net/publication/49606938_CONCIENCIA_AMBIENTAL_EN_ESTUDIANT ES_DE_ENFERMERIA_BASICA 